\title{
Cosmic ray modulation by corotating interaction regions
}

\author{
Jaša Čalogović ${ }^{1}$, Bojan Vršnak ${ }^{1}$, Manuela Temmer ${ }^{2}$ and \\ Astrid M. Veronig ${ }^{2}$ \\ ${ }^{1}$ Hvar Observatory, Faculty of Geodesy, Kačićeva 26, HR-10000 Zagreb, Croatia \\ ${ }^{2}$ Institute of Physics, University of Graz, Universitätsplatz 5, A-8010 Graz, Austria \\ email: jcalogovic@geof.hr
}

\begin{abstract}
We analyzed the relationship between the ground-based modulation of cosmic rays $(\mathrm{CR})$ and corotating interaction regions (CIRs). Daily averaged data from 8 different neutron monitor (NM) stations were used, covering rigidities from $R_{c}=0-12.91 \mathrm{GeV}$. The in situ solar wind data were taken from the Advanced Composition Explorer (ACE) database, whereas the coronal hole $(\mathrm{CH})$ areas were derived from the Solar X-Ray Imager onboard GOES-12. For the analysis we have chosen a period in the declining phase of solar cycle 23 , covering the period 25 January-5 May 2005. During the CIR periods CR decreased typically from $0.5 \%$ to $2 \%$. A cross-correlation analysis showed a distinct anti-correlation between the magnetic field and $\mathrm{CR}$, with the correlation coefficient $(r)$ ranging from -0.31 to -0.38 (mean: -0.36 ) and with the CR time delay of 2 to 3 days. Similar anti-correlations were found for the solar wind density and velocity characterized by the CR time lag of 4 and 1 day, respectively. The relationship was also established between the CR modulation and the area of the CIR-related $\mathrm{CH}$ with the $\mathrm{CR}$ time lag of 5 days after the central-meridian passage of $\mathrm{CH}$.
\end{abstract}

Keywords. (ISM:) cosmic rays, (Sun:) solar wind, (Sun:) solar-terrestrial relations

\section{Introduction}

Corotating interaction regions (CIRs) are regions of compressed plasma formed at the leading edges of corotating high-speed solar wind streams originating in coronal holes (CHs) as they interact with the preceding slow solar wind. CIRs and high speed streams modulate galactic cosmic rays (GCR), thus influencing the flux of cosmic rays (CR) at the Earth (e.g. Richardson 2004). With the aim to improve space weather predictions, we investigated the relationship between CR and CIR.

\section{Data and method}

The daily averaged count rates from 8 different NM stations were used, supplied by SPIDR website (http://spidr.ngdc.noaa.gov/spidr). NM stations were selected to cover all latitudes and longitudes (3 NM stations in high, 3 in middle and 2 in low latitudes; see Table 1) with the cut-off rigidities ranging from $R_{c}=0-12.91 \mathrm{GeV}$.

Given that high solar activity and CMEs strongly affect CR, we avoided a period with CME activity. A period close to the declining phase of solar cycle 23 was selected, covering 25-125 (25 January until 5 May) days of the year (DOY) 2005. In this period the solar CME activity was particularly low (Vršnak et al. 2007a).

The data on proton density $n$, proton speed $v$, and magnetic field strength $B$ were taken from the Advanced Composition Explorer (ACE) database (Stone et al. 1998). Daily solar coronal hole areas were determined employing the data from Solar X-Ray 


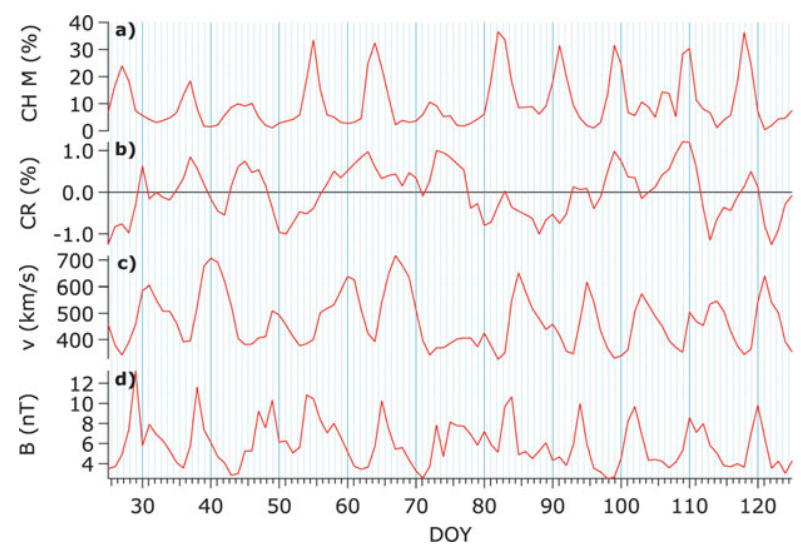

Figure 1. a) Daily measurements of the $\mathrm{CH}$ fractional area in the $\mathrm{M}$ slice, b) Relative cosmic ray change measured with Climax neutron monitor $\left(R_{c}=3.03 \mathrm{GeV}\right)$, c) ACE daily averages of the proton velocity $v$, and d) magnetic field strength $B$. The $x$-axis represents DOY for 2005 .

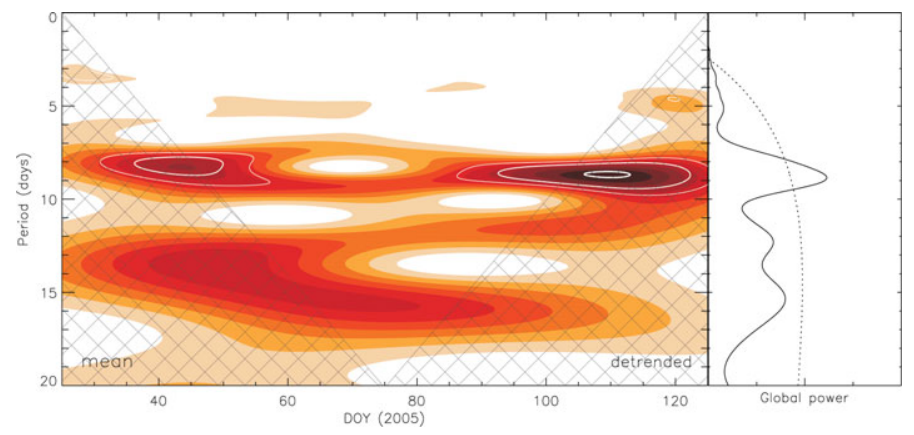

Figure 2. Averaged WPS (left) and GWS (right) for all 8 NM stations together. Analyzed time span is from 25 January 2005 until 5 May 2005 (DOY 25-125). The cone of influence is indicated by cross-hatched regions. With the white contour lines are marked the significance levels of $70 \%$ (thin white line), $80 \%$ (thicker white line) and $90 \%$ (the thickest white line). The dotted line in the GWS represents the $95 \%$ significance level.

Imager (SXI) on board the GOES-12 satellite, where the coronal holes appear as dark features in X-ray images (Hill 2005; Pizzo 2005). Using one SXI level-2 image per day, fractional areas of coronal holes were extracted in three meridional slices in the longitude range: $\mathrm{CH} \mathrm{E}\left[-40^{\circ}-20^{\circ}\right], \mathrm{CH} \mathrm{M}\left[-10^{\circ} 10^{\circ}\right.$ ] (Fig. 1a) and $\mathrm{CH} \mathrm{W}\left[20^{\circ} 40^{\circ}\right]$, for details see Vršnak et al. (2007b).

With an emphasis on daily variations in the CR, the NM data were detrended. The detrended NM data were cross-correlated with the $\mathrm{CH}$ areas and solar wind data, allowing for time lags of up to 10 days. The statistical significance was tested using one sided t-test on $5 \%$ level.

To determine the predominant periods in the NM data, as well as their times of appearance, wavelet power spectra (WPS) and global wavelet spectra (GWS) were used (Morlet wavelet; see Temmer et al. 2007).

\section{Results}

In Fig. 1a the $\mathrm{CH}$ fractional area for the central meridian slice is presented as a function of DOY 2005. Oscillations of the $\mathrm{CH}$ area show the most prominent period of about 9 


\begin{tabular}{l|cccccc}
\hline & CH E & CH M & CH W & $v$ & $B$ & $n$ \\
\hline NM South Pole & $-0.33(6)$ & $-0.31(4)$ & $-0.30(3)$ & $-0.21(0)$ & $-0.31(2)$ & $-0.24(4)$ \\
NM Thule & $-0.16^{*}(7)$ & $-0.20(4)$ & $-0.14^{*}(3)$ & $-0.09^{*}(0)$ & $-0.37(2)$ & $-0.23(4)$ \\
NM Magaadan & $-0.34(7)$ & $-0.33(5)$ & $-0.34(3)$ & $-0.24(0)$ & $-0.33(2)$ & $-0.20(4)$ \\
NM Jungfraujoch & $-0.31(8)$ & $-0.26(5)$ & $-0.32(4)$ & $-0.21(2)$ & $-0.35(3)$ & $-0.22(4)$ \\
NM Climax & $-0.34(7)$ & $-0.31(5)$ & $-0.31(3)$ & $-0.20(1)$ & $-0.38(3)$ & $-0.33(4)$ \\
NM Hermanus & $-0.27(7)$ & $-0.27(5)$ & $-0.27(3)$ & $-0.11^{*}(1)$ & $-0.38(3)$ & $-0.32(4)$ \\
NM Haleakala & $-0.23(7)$ & $-0.26(5)$ & $-0.32(3)$ & $-0.20(1)$ & $-0.34(3)$ & $-0.16^{*}(4)$ \\
NM Tibet & $-0.34(7)$ & $-0.39(5)$ & $-0.41(3)$ & $-0.27(1)$ & $-0.38(3)$ & $-0.18(4)$ \\
average & $-0.29(7.00)$ & $-0.29(4.75)$ & $-0.30(3.13)$ & $-0.19(0.75)$ & $-0.36(2.63)$ & $-0.24(4.00)$ \\
$\sigma$ & 0.065 & 0.056 & 0.078 & 0.062 & 0.026 & 0.063 \\
\hline
\end{tabular}

Table 1. Correlation coefficients between the CR measured at 8 different NM stations and $\mathrm{CH}$ areas (three meridional slices in the longitude range: east - $\mathrm{CH} \mathrm{E}$, middle - $\mathrm{CH} \mathrm{M}$, west - $\mathrm{CH}$ $\mathrm{W}$ ), magnetic field $B$, proton speed $v$ and density $n$. The highest correlation coefficient in the 10-days lag period is shown. Numbers in brackets indicate corresponding lag in days. All NM data are detrended. The non-significant values are marked with * .

days (Temmer et al. 2007), which can be explained by a "triangular" distribution of large $\mathrm{CHs}$, separated by $\approx 120^{\circ}$ in longitude. Similar oscillations we find also in the Climax NM data (Fig. 1b), as well as in the solar wind parameters data (proton speed $v$ in Fig. 1c and magnetic field $B$ in Fig. 1d). During the CIR passage the CR decreased typically from $0.5 \%$ to $2 \%$ (Fig. $1 \mathrm{~b}$ ). Figure 2 shows the detected periods in the CR data using a Morlet wavelet, where the most prominent period is also in the range of 9 days (DOY 30 - 125) and slightly weaker periods are in the range of 13.5 days (DOY $20-65$ ) as well as 12 and 15 days (DOY $65-115$ ).

The results of a cross-correlation analysis are presented in Table 1 for each NM station, where the highest correlation coefficients $(r)$ within a 10-day lag period are shown. First three columns reveal a distinct anti-correlation (mean $r=-0.29$ ) between the area of the CIR-related $\mathrm{CH}$ and the $\mathrm{CR}$ modulation. Depending on the $\mathrm{CH}$ slice, $\mathrm{CR}$ show time lag of 7,5 , and 3 days for the east, middle and west $\mathrm{CH}$ slice, respectively. A significant relationship was also established between $\mathrm{CR}$ and solar wind parameters: $v, B$ and $n$ (Table 1). The highest correlations with $\mathrm{CR}$ was obtained for the magnetic field $B$ with the CR time delay of 2 to 3 days, followed by proton density $n$ (time lag 4 days) and proton velocity $v$ (time lag $0-1$ day).

\section{Conclusions}

The magnetic field enhancement forming at frontal edge of high-speed streams in the solar wind acts as a shield that reduces GCR flux at $1 \mathrm{AU}$ by $0.5 \%-2 \%$. The effect is especially prominent during the declining phase of the solar cycle, when the occurrence rate of equatorial $\mathrm{CHs}$ is increased and the $\mathrm{CME}$ activity is low. A typical lag between the magnetic field peak and the CR dip is $2-3$ days.

\section{References}

Hill, S. M. et al. 2005, Solar Phys., 226, 255

Pizzo, V. J. et al. 2005, Solar Phys., 226, 283

Richardson, I. G. 2004, Space Sci. Revs, 111(3), 267

Stone, E. C., Frandsen, A. M., Mewaldt, R. A., Christian, E. R., Margolies, D., Ormes, J. F., \& Snow, F. 1998, Space Sci. Revs, 86, 1

Temmer, M., Vrsnak, B., \& Veronig, M. A. 2007, Solar Phys., 241, 371

Vršnak, B., Temmer, M., \& Veronig, M. A. 2007a, Solar Phys., 240, 331

Vršnak, B., Temmer, M., \& Veronig, M. A. 2007b, Solar Phys., 240, 315 